\title{
Platelet-Rich Plasma in Treatment of Plantar Fasciitis: Randomized Double-Blinded Placebo Controlled Study
}

\author{
Akram Abdel Moneim Deghady, MD ${ }^{1 *}$; Mowaffak Moustafa Abdel Hamid, MD²; \\ Abdel Moneim Hussein Helal, MD²; Sherine Mahmoud El-Sherif, MD²; \\ Hadeer Al Sayed Abdel Latief, MS²
}

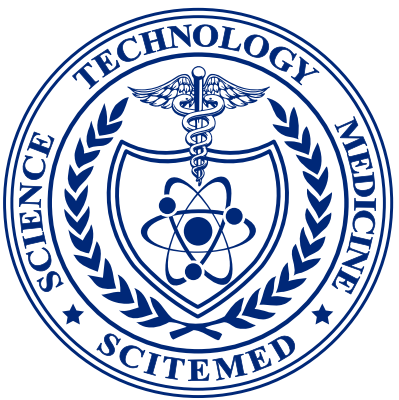

\author{
Department of Clinical and Chemical Pathology, Faculty of Medicine, Alexandria University, Alexandria, Egypt \\ 2 Department of Physical Medicine, Rheumatology and Rehabilitation. Faculty of Medicine, Alexandria University, Alexandria, Egypt
}

\begin{abstract}
Objective: Plantar fasciitis is one of the most common causes of heel pain. Conservative noninvasive lines of treatment are numerous, among which platelet-rich plasma (PRP) has recently been demonstrated to be helpful in managing plantar fasciitis. The aim of this study is to evaluate the platelet-rich plasma-ultrasound-guided injection in the treatment of plantar fasciitis through randomized double-blinded placebo-controlled study.

Methods: Sixty-two adult plantar fasciitis patients were involved in the current study. They were randomly selected from the outpatient clinics of Physical Medicine, Rheumatology and Rehabilitation, Faculty of Medicine, Alexandria University. They were randomly divided to receive either saline (group 1) or PRP (group 2) injection under ultrasonography (US)-guidance. Assessment was done using visual analogue scale (VAS) for pain intensity at baseline and during $2^{\text {nd }}$ and $6^{\text {th }}$ weeks after injection. Functionally, foot function index (FFI) was used at baseline and during $6^{\text {th }}$ week after injection. Pain relief score (PRS) was measured during $2^{\text {nd }}$ and $6^{\text {th }}$ weeks after injection. Plantar fascia thickness was also measured sonographically by US at baseline and during $2^{\text {nd }}$ and $6^{\text {th }}$ weeks after injection.

Results: There was no statistically significant difference between both groups as regards age, Body Mass Index and baseline US, VAS, and FFI scores. PRP (group 2) showed significant improvement in pain severity and physical limitations in patients with plantar fasciitis. It also showed significant improvement compared to saline group injection considering VAS, US, PRS, and FFI.

Conclusion: The use of PRP injection for treatment of plantar fasciitis resulted in significant improvement in pain according to VAS, PRS, and FFI and a reduction in the thickness of the plantar fascia as measured by US, compared to the saline group.
\end{abstract}

\section{Introduction}

Plantar fasciitis is one of the most common causes of foot conditions $[1,2]$. The peak age of incidence is between $40-60$ years [3,4]. Repetitive microtrauma arising from heel strike leads to traction periostitis and results in inhibition of normal repair process that leads to chronic inflammation of the fascia $[1,5]$.

The risk factors for developing plantar fasciitis are excessive foot pronation, high arched foot, leg length discrepancy, and overweight individuals who spend long periods standing $[6,7]$. Diagnosis of plantar fasciitis is based on the patient's history and physical examination. The patient feels initial pain on the first step out of bed, which is relieved with gradually increased activity and elicited on palpation of the medial plantar calcaneal region $[1,5,8]$.

Plantar fasciitis is a self-limiting condition, but complete resolution may take 3 to 18 months or longer, which impacts the quality of life [9]. Conservative noninvasive lines of treatment are numerous, among which platelet-rich plasma (PRP) is a recent and promising modality $[8,10]$. PRP is a platelet-rich concentrate with platelet level greater than the baseline by several folds $[11,12]$. It modulates collagen synthesis, decreases inflammation, promotes tissue healing, and stimulates fibroblast activity $[13,14]$. PRP has high levels of platelets and a full complement of clotting and growth factors [15]. There is an increasing interest in PRP as a treatment for plantar fasciitis, and recently several papers on this topic have been published $[16,17]$. The aim of this study was to evaluate the value of PRP in the management of plantar fasciitis through randomized double-blinded placebo-controlled study, as there was a debate on the value of using PRP in such condition $[18,19,20]$.

\section{Methods}

All the patients attending the Physical Medicine, Rheumatology and Rehabilitation clinic of Alexandria Main University Hospital with plantar fasciitis from September 2016 to August 2017 that fulfilled the inclusion criteria, which were patients aged $>18$ years and having a score of 5 or more on the visual analogue pain scale (VAS), were included in the study [17].

There were total 74 patients. The patients were excluded if they received local steroid injection within 1 month, physical therapy within 6 weeks, non-steroidal anti-inflammatory drugs or acetylsalicylate within 1 week, and had undergone previous surgery for plantar fasciitis, or had history of foot fracture. Besides, those patients with diabetes mellitus, rheumatoid arthritis, gout, hypothyroidism or other painful or function limiting disorders of the foot and ankle, presence of severe anemia, platelet count $<100,000$ per microliter of blood, significant cardiovascular, and renal or hepatic disease were also excluded from the study. Seven patients did not meet our inclusion criteria ( 4 had gout and 3 were diabetic) and 5 patients were lost in follow-ups and we had excluded them from the study.

In this study, the plantar fasciitis patients were allocated by a trained nurse into two equal groups of 31 patients each by simple randomization method. Patients were blinded to the therapy received as well as the assessors (the assessors giving the injectable and the assessors performing the ultrasonography). Both groups were taken by the nurse to the department of clinical pathology to withdraw a blood sample for each. After a period of time, the nurse returned to collect the prepared samples, whether PRP or saline. Then she prepared the syringes for injection after labeling it with a white paper showing name code of the patients and adding calcium for activation. At the end of the study, the trained nurse uncoded the two groups of patients in presence of the assessors. 
The injection procedure was sonographically guided at the physical medicine, rheumatology and rehabilitation clinic of Alexandria University Hospitals. Approval for this prospective clinical study was granted by the local ethics committee and an informed consent was obtained from all patients participating in the study. All patients were subjected to the following: (1) Assessment of foot function using the foot function index (FFI) initially and at 6 weeks follow-up [21]. FFI is a questionnaire designed to give information as to how the foot pain has affected the patient's ability to manage in everyday life. Each question was scored on a scale from 0 (no pain) to 10 (worst pain imaginable) that best described the foot over the last week. High scores correlated with poor foot function [21]. (2) The pain intensity was assessed with VAS initially and during $2^{\text {nd }}$ and $6^{\text {th }}$ weeks after injection (Figure 1) [17]. (3) Pain relief score (PRS) was measured during $2^{\text {nd }}$ and $6^{\text {th }}$ weeks after injection. The score varied from 0 (no relief), 1 (slight relief), 2 (moderate relief), 3 (good relief), to 4 (complete relief). (4) Ultrasonography (US) examination was performed using a 10 $\mathrm{MHz}$ linear array transducer (Siemens-Germany) initially and during $2^{\text {nd }}$ and $6^{\text {th }}$ weeks after injection. Increased plantar fascia thickness greater than $0.4 \mathrm{~cm}$ was considered as a diagnostic sonographic finding in plantar fasciitis [22,23]

We have used the endpoints of $2^{\text {nd }}$ and $6^{\text {th }}$ weeks in the follow-ups. At the acute inflammatory phase, the motion is limited until the inflammation subsides. Our patients started stretching exercises for 2 weeks post-injection and this was followed by the first follow-up [17,24]. Then, at the $4^{\text {th }}$ week post-injection, the patients were allowed to start their recreational activities and this was followed 2 weeks later by the second follow up (which was at the $6^{\text {th }}$ week) $[25,26]$.

\section{Platelet-Rich Plasma Preparation Method}

Two whole blood samples each $(9 \mathrm{ml})$ were obtained by venipuncture in acid citrate dextrose (ACD) $1 \mathrm{ml}$ in a sterile vacutainer. The blood was centrifuged using a 'soft' spin $(250 \mathrm{~g})$ for 10 minutes (Hettich zentrifugen portofix 32 A, Germany). The supernatant plasma containing platelets was transferred into another sterile tube without anticoagulant (Lab Companion, Korea). The tube was centrifuged at a higher speed (300 $\mathrm{g}$ for 10 minutes) to obtain a platelet concentrate. At the bottom of the tube, platelets pellet was formed. Platelet-poor plasma (PPP) was removed and the platelet pellet was suspended in $2 \mathrm{~mL}$ PPP by vortex (Vortex Mixer, Taiwan). The PRP was mixed with $10^{\text {th }}$ the volume of calcium gluconate and injected.

\section{Sonographically Guided Injection}

Patients were placed in a prone position with the feet hanging over the edge of the examination table, with dorsiflexion of the toes to stretch the plantar fascia so that its margins were seen clearly. Longitudinal images of the plantar fascia close to the calcaneal enthesis were obtained and fascial thickness was measured. The thickness of the plantar fascia was measured 10 to $15 \mathrm{~mm}$ from the insertion of the calcaneus at its thickest portion in each patient. A 4-cm 21-gauge needle was inserted perpendicular to the long axis of the ultrasound transducer into the inflamed portion of the plantar fascia from its attachment to the calcaneus. The needle was withdrawn to the near edge of the fascia under sonographic guidance, enabling dispersion of the injected solution around the edge. This sonographic assessment was repeated later during $2^{\text {nd }}$ and $6^{\text {th }}$ weeks for follow-up.

\section{Statistical Analysis}

Using PASS 2000 software, a sample size of 62 (31 per group) achieved $80 \%$ power to detect a difference between the two groups in VAS, at a significance level (alpha) of .05 using a two-sided Mann-Whitney test.

After the collection of data, it was coded and transformed into a specially designed format suitable for computer feeding. All the entered data were verified for error. Data were analyzed using the Statistical Package for Social Sciences (SPSS ver.20 Chicago, IL, USA).

The distribution of quantitative variables was tested for normality using the Kolmogorov-Smirnov test. They were not normally distributed, and were described using median and range and represented by box plots. The blue, green, and yellow boxes are the scores between $25^{\text {th }}$ and $75^{\text {th }}$ percentile, the upper border represents the upper quartile range, and the lower border represents the lower quartile range. The vertical bars include all the samples, with the exception of the scores, accounted as outliers and plotted as dots (outliers are data that are far from the upper border or lower border of the box by about 1.5 interquartile range), and extremes are plotted as stars (extremes are data that are away from the upper border or lower border of the box by 3 times interquartile range). The upper horizontal bar represents the maximum, the lower horizontal bar represents the minimum, and the wide line represents the median range.

Mann Whitney $U$ test was used to compare quantitative variables between two groups. Wilcoxon Signed-rank test was used to compare quantitative parameters at 2 intervals in the same group. Friedman ANOVA test was used to compare quantitative parameters at 1 week, 2 weeks, and 6 weeks in the same group, and if significant, it was followed by pairwise comparison. Spearman correlation test was used to correlate between quantitative variables. In all statistical tests, the level of significance of .05 was used, below which the results were considered to be statistically significant.

\section{Results}

The aim of this study was to evaluate the PRP ultrasound-guided injection in the treatment of plantar fasciitis. There was no statistically significant difference at baseline between both groups regarding demographic data and other clinical and sonographic measures, i.e., the thickness of tendons by US, VAS, and FFI as shown in Table 1. There was a statistically significant improvement in both groups regarding FFI and PRS as presented by box plots in Figure 2 and Figure 3. There was a statistically significant improvement in both groups regarding sonographic plantar fascia thickness and VAS at baseline, first, and second follow up (Table 2).

Both groups were then compared to assess the superiority of PRP over placebo. Table 3 presents the percent change of outcome parameters calculated between the initial assessment and the final one. Percent change in plantar fascia thickness was significantly higher in the PRP group than in the saline group. Percent change in VAS score of pain was significantly higher in the PRP group than in the saline group. Also, percent change in FFI was significantly higher in the PRP group than in the saline group. There was no statistically significant difference in percent change regarding PRS.

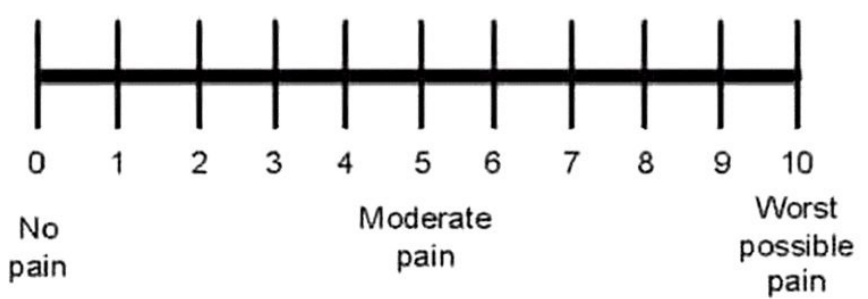

Figure 1. Visual analogue scale pain intensity score.

\section{Discussion}

Plantar fasciitis is the most common cause of heel pain [1,2]. It has been attributed to many different etiological factors $[6,7]$. There are many available treatment methods, but the unsatisfactory outcome of the treatment modalities leads the patient to seek a more recent effective modality $[8,10]$. 


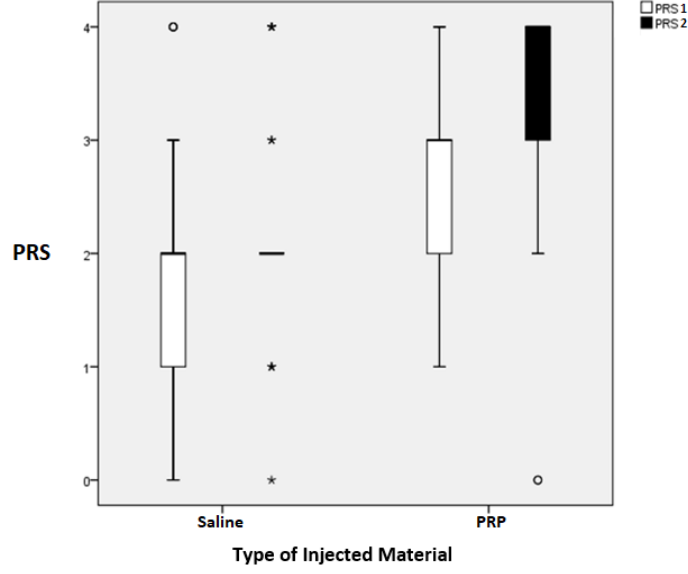

Figure 2. Box plot for comparison between the two groups at baseline and $6^{\text {th }}$ week after injection as regard PRS. Upper horizontal bar represents the maximum. Lower horizontal bar represents the minimum. The wide line represents the median range. Outliers plotted as dots (data that are far from the upper border or lower border of the box by about 1.5 interquartile range). Extremes plotted as stars (data that are away from the upper border or lower border of the box by 3 times interquartile range). PRS, pain relief score; PRS 1, pain relief score at $2^{\text {nd }}$ week follow-up; PRS 2 , pain relief score at $6^{\text {th }}$ week follow-up.

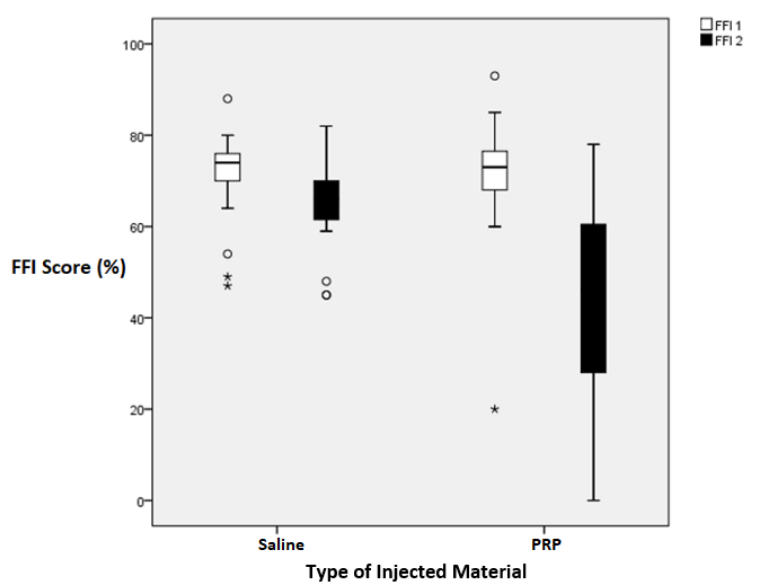

Figure 3. Box plot for comparison between the two groups at $2^{\text {nd }}$ and $6^{\text {th }}$ week after injection as regard FFI. Upper horizontal bar represents the maximum. Lower horizontal bar represents the minimum. The wide line represents the median range.Outliers plotted as dots (data that are far from the upper border or lower border of the box by about 1.5 interquartile range). Extremes plotted as stars (data that are away from the upper border or lower border of the box by 3 times interquartile range). FFI, foot function index; FFI 1, foot function index initially; FFI 2, foot function index at $6^{\text {th }}$ week follow-up.

\begin{tabular}{|c|c|c|c|}
\hline & Saline, $N=31$ & $P R P, N=31$ & $P$ \\
\hline Age, years (range) & $42(22-60)$ & $40(27-69)$ & 0.916 \\
\hline BMI, kg/m² (range) & $29(21-40)$ & $29(24-45)$ & 0.444 \\
\hline Disease duration, months (range) & $12(1-120)$ & $9(1-72)$ & 0.470 \\
\hline VAS (range) & $9(7-10)$ & $9(6-10)$ & 0.842 \\
\hline US, mm (range) & $5(3-6)$ & $5(3-6)$ & 0.797 \\
\hline FFI, \% (range) & $74(47-88)$ & $73(20-93)$ & 0.843 \\
\hline
\end{tabular}

The data were presented in median. BMI, body mass index; FFI, foot function index; PRP, platelet-rich plasma; US, ultrasonography; VAS, visual analogue scale.

\begin{tabular}{|c|c|c|c|c|c|c|}
\hline & & $\mathrm{N}$ & Baseline & $1^{\text {st }}$ Follow-up & $2^{\text {nd }}$ Follow-up & $\mathrm{P}$ \\
\hline \multirow{2}{*}{ US (mm) } & Saline group (range) & 31 & $4.8(3-6)$ & $4.4(2-6)$ & $4.3(2-6)$ & $<0.001$ \\
\hline & PRP group (range) & 31 & $4.9(3-6)$ & $4.4(2-5)$ & $4(2-6)$ & $<0.001$ \\
\hline \multirow{2}{*}{ VAS } & Saline group (range) & 31 & $9(7-10)$ & $7(2-10)$ & $7(0-10)$ & $<0.001$ \\
\hline & PRP group (range) & 31 & $9(6-10)$ & $5(0-9)$ & $4(0-9)$ & $<0.001$ \\
\hline
\end{tabular}

All groups were significantly different from each other by pair wise comparison. The data were presented in median. PRP, platelet-rich plasma; US, ultrasonography; VAS, visual analogue scale.

\begin{tabular}{|c|c|c|c|}
\hline & Saline, N=31 & $P R P, N=31$ & $P$ \\
\hline US change, \% (range) & $16.67(0-33.33)$ & $20(-20-60)$ & 0.050 \\
\hline VAS change, $\%$ (range) & $22.22(0-100)$ & $60(0-100)$ & $<0.050$ \\
\hline PRS change, \% (range) & $0(-50-100)$ & $33.33(-100-300)$ & 0.550 \\
\hline FFI change, \% (range) & $8.57(-25.53-37.5)$ & $34.12(-65-100)$ & $<0.050$ \\
\hline
\end{tabular}

The data were presented in median. FFI, foot function index; PRP, platelet-rich plasma; US, ultrasonography; VAS, visual analogue scale. 
PRP is a new modality in the treatment of plantar fasciitis. Our study was designed to compare the effect of PRP injection with saline injection in the treatment of plantar fasciitis, both clinically and sonographically, within 6 weeks after the injection.

Our results showed that both groups had statistically significant clinical and sonographic improvement at the end of the 6 weeks follow-up period without any complications, and it is greater in the PRP than in the saline group. This improvement included VAS, PRS, and FFI scores. The effect of PRP on healing in plantar fasciitis is mainly related to platelet-derived growth factor (PDGF), platelet-derived endothelial growth factor (PDEGF), transforming growth factor (TGF), Interleukins 4,8,13,17, tumor necrosis factor alpha, and interferon alpha [27]. This combination of growth factors and anti-inflammatory and pro-inflammatory cytokines present in the platelet $\propto$-granule have been shown to initiate the healing stages necessary to reverse the degenerative process at the base of the plantar fascia, enhance fibroblast migration and proliferation, increase vascularization, increase collagen deposition, removal of tissue debris, angiogenesis and laying extracellular matrix [17,19,20]. Transforming growth factor beta one (TGF-B1) is shown to significantly increase type I collagen production by tendon sheath fibroblasts [17].

In agreement with our study, Gupta et al. [18] and El Mallah et al. [19] reported that PRP injection was a promising safe line of treatment for chronic plantar fasciitis, carrying no complications, effective in relieving pain and improving function. On the other hand, de Vos et al. [20] randomly studied the effect of injection of platelet-rich plasma in chronic tendinopathy. They concluded that among the patients with chronic tendinopathy, a PRP injection compared with a saline injection did not result in a greater improvement in pain and activity. Therefore, they did not recommend this treatment in chronic tendinopathies including plantar fasciitis. They attributed both treatment groups' clinical improvement to the eccentric exercises which were performed and proved to improve pain and function. Another explanation was the length of time the platelets remained at the site after injection in the degenerated area. Platelets required time to be slowly activated by exposure to collagen. It might be due to the pressure within the tendon that a large amount of PRP diffused rapidly, thereby reducing its effect. Sheth et al. [27] studied the efficacy of autologous PRP use for orthopedic indications. They concluded that there was uncertainty about the evidence to support the increasing clinical use of PRP as a treatment modality for orthopedic bone and soft tissue injuries. This could be explained by a lack of standardization of study protocol, platelet separation techniques, and outcome measures.

In the present study, the percent change reflecting a reduction in the plantar fascia thickness was significantly higher in the PRP group than in the saline group. Ultrasound has proven to be a useful tool to assess plantar fasciitis through the production of high-quality spatial resolution sonograms [28].

In general, US could demonstrate a reduction of plantar fascia thickness with the PRP group injection in patients with plantar fasciitis [17]. There is agreement among authors that US is a valuable noninvasive imaging modality for diagnosis of plantar fasciitis and in the follow-up assessment as it is free of the hazardous ionizing radiation $[16,17]$. It doesn't affect the biomechanical function of the foot, it is inexpensive and portable, and it allows real-time imaging [16,17].

In the present study, US has been used to guide injection procedure following emerged recommendations by published studies. Patients treated by ultrasound-guided injection tended to suffer less pain and achieved a higher response rate [29]. Hamayouni et al. [30] reported that ultrasound-guided injection can help with the reduction in plantar fascia thickness and pain. The use of ultrasound as a procedure guiding technique provides real-time monitoring at the needle tip allowing for precise needle placement in the target, instantly saving the nearby important structures from unnecessary puncture or injury. Moreover, watching the needle tip ensures that the needle tip does not extend outside the lesion [16]. It is reasonable that the ultrasound-guided injection achieves better outcomes. In recognition of these advantages, the use of US-guided injection technique in the present study proved to increase the accuracy of injection, site localization, speed-up procedure and minimize non-target tissue damage.
On the other hand, several studies suggested that non-ultrasound guided injection was cheaper and equally effective to ultrasound-guided injection [31]. Kane et al. [32] reported no significant difference in their comparative study between ultrasound-guided and palpation-guided injection techniques in the management of idiopathic plantar fasciitis.

In our study, the saline group also showed statistically significant improvement as regards outcome measures. This can be attributed to the needling effect of the injection [33,34]. Needling consists of multiple tendon perforations without injecting any substances [33]. It has been suggested that the beneficial effect of needling results from improved muscle activation pattern, increased joint range of motion, and alleviation of pain [35]. In the context of plantar fasciitis, needling may be responsible for the central release of opioid peptides, increased regional blood flow and anti-inflammatory effects presumably through inhibition of production of tumor necrosis factor-alpha, interleukin-1 beta, and interleukin-6 $[28,36]$. Rastegar et al. [36] compared the effect of needling and steroid injection in the treatment of plantar fasciitis. They found that needling was superior to steroid injection in patients with plantar fasciitis in the long term. On the other hand, Cotchett et al. [37] found a statistically significant improvement in plantar heel pain after needling, but warned about some complications such as infection, fever, and tissue hypertrophy. The outcome of PRP injection may vary in relation to several factors such as centrifugation, technique, activating agents, platelet count, and the presence of leukocytes in the prepared PRP.

Centrifugation is a simple technique and may yield platelet concentrates three to eight-fold higher than whole blood [38]. Centrifugation has two parameters that may influence the outcome of platelet concentrate. The first is whether it is done once (single spin) or twice (double spin). The second is the centrifugation speed. In the present study, the blood was centrifuged using a double centrifugation (the first spin $250 \mathrm{~g}$ for 10 minutes and the second spin from $300 \mathrm{~g}$ for 10 minutes), where $\mathrm{g}=$ relative centrifugation force.

Researchers advocate a double-spinning technique instead of a single-spinning method because the former generates a higher platelet concentration of $6 x$ to $9 x$ and thus results in better efficacy of the final PRP product $[39,40]$. In a study, a double centrifugation technique was performed in plantar fasciitis. The resulting platelets concentrate contained approximately 6-8 times the concentration of platelets compared to baseline whole blood. They found that the VAS score decreased from 9.1 prior to the injection to 1.6 following the application. The rate of patient satisfaction was $88 \%$ and significant changes in the thickness of the plantar fascia were observed during the study period [17]. However, in other studies, variations in platelet concentrate resulting by double-spin centrifugation had been encountered. In one study, this centrifugation technique yielded 5 times the concentration and another yielded 3-5 times the concentration $[18,41]$.

On the other hand, Filardo et al. [42] compared the acquisition of platelets concentrates by single or double centrifugation. They found that the benefit was similar in both groups. However, adverse events (especially local arthritis) occurred more often in the PRP obtained by double centrifugation, due to higher leukocyte concentration produced by this method [42]. Regarding the centrifugation speed, Bausset et al. [43] observed that a centrifugation of $400 \mathrm{~g}$ or $1000 \mathrm{~g}$ did not increase the platelet concentration compared to $250 \mathrm{~g}$ centrifugation. This could be due to platelet aggregation, which indicated that centrifugation at higher speeds was not suitable for PRP preparations as it could be deleterious for the platelet function. They also found that high centrifugation speeds modified the morphology of the platelets, thereby proving that lower speeds were better suited for the preservation of the platelet morphology.

Some authors recommend the single spin centrifugation as it generates forces that are at least 3 times less harmful to the platelets [40]. They ascribed that the increase in platelet concentration over $59 \%$ of the whole blood might be attributed to the replacement of the blood cell volume with plasma and platelets during the low-speed centrifugation [40].

To sum up, there is no solid agreement on the preparation method for obtaining an efficient PRP sample that induces better clinical respons- 
es. So, there may be other factors that influence the efficacy of the prepared PRP.

Calcium did not result in any of the disadvantages of thrombin that was previously considered to activate platelets [41]. Calcium gluconate ampoules (Calcionate-Memphis-Egypt) as a source of calcium were found to offer better sterilization condition than calcium chloride. The advantages of activation of platelets with calcium included general release of growth factors over time, with a lower initial level followed by a progressively increasing amount of growth factors released, reaching higher levels at the 24-hour evaluation [44]. Also, it delayed clot formation, no formation of antibodies, an incidence that was associated with the use of bovine thrombin as activator $[44,45]$.

For autologous PRP preparation, the platelet count in the produced PRP will certainly depend on whole blood platelet count. Patients with low platelets count will have autologous PRP with relatively low count as well. Since the number of growth factors released depend on the number of activated platelets in the final injected PRP, it is reasonable to think that the PRP efficiency will be significantly lowered if prepared for patients with low platelet count. In addition, PRP platelet count will also depend on the preparation method, specifically the centrifugation, as previously explained. Consequently, it may be consistent to consider the post-preparation count of platelets to ensure that the preparation methods are reliable in producing the target platelet concentrate.

In this regard, in the present study, all subjects had whole blood platelets count within the normal range. As the double-spin centrifugation used in this study was expected to yield PRP with approximately 4-6 folds [29,3], it could be assumed that the PRP prepared for injection had sufficient platelet count to release effective growth factors. However, this remains argumental without precise knowledge of the count of platelets present actually in the final PRP preparation.

There is no agreement in the literature about the effective platelet count for effective PRP sample. Some authors used a high concentration of platelets up to 6-8 folds compared to whole blood [46]. Others found $\geq 4 \times[30,39]$ or as low as $2.5 \times[47]$ to be effective. Such variations in reporting on the effective concentration of platelets in PRP may be due to the variability of the targeted pathologies. Alternatively, it may reflect the multifactorial background that determines the effectiveness of PRP.

The influence of the presence of leukocytes contained in the prepared PRP on the final outcome is controversial. These cells contain anti-inflammatory and pro-inflammatory cytokines that can affect the final outcome differently. While the former can help prevent infection $[44,48]$, the latter may trigger local inflammatory reactions and enhance pain $[42,49]$. Accordingly, there seems to be no agreement on whether to use PRP low in leukocytes or not. In the present study, care was exercised to avoid inclusion of leukocytes in the final PRP preparation. It could be assumed that the used preparation contained low leukocyte count as in none of the studied cases was there any signs of local pain or inflammation.

The use of local anesthesia has been found to contribute negatively to the outcome [50]. On one hand, it may lead to effect overlap [51]. On the other hand, local anesthetics are probably responsible for reducing the effect of PRP by shifting $\mathrm{pH}$ to acidity detrimental to treated cells [50]. Nevertheless, local anesthetics are found to negatively influence tenocyte in proliferation and viability [50]. However, and on the positive side, local anesthetics are found not to suppress the release of growth factors [52].

In the present study, we have avoided all the negative effects of local anesthetics by not using any. Despite not using local anesthetics in our patients, none has reported any significant discomfort during or after the injection procedure.

A limitation in our study was that we did not standardize the number of platelets each time for injection. So, the number of platelets injected was dependent on the original platelets count in each patient blood. We did not have a relation between the PRP count and the efficacy of treatment. Finally, we did not compare PRP with other treatment modalities e.g., corticosteroids. Our point of strength in this study, basically, was complete blindness of the procedure. We have not included any disease that interferes with the platelet action. Finally, there was no treatment taken by our patients during the period of the study e.g., nonsteroidal anti-inflammatory drugs.

\section{Conclusion}

In conclusion, the use of PRP injection for treatment of plantar fasciitis resulted in significant improvement in pain according to VAS, PRS, and FFI and a reduction in the thickness of the plantar fascia as measured by US compared to the saline group.

\section{Article Information}

* Correspondence: Akram Abdel Moneim Deghady, MD

Department of Clinical and Chemical Pathology, Faculty of Medicine, Alexandria University, 201 Port Said street, Sporting, Alexandria, Egypt.

E-mail: akram61@yahoo.com

Received: Apr. 24, 2018; Accepted: Jun. 20, 2018; Published: Feb 09, 2019

\section{DOI: 10.24983/scitemed.jacp.2019.00097}

Copyright @ 2019 The Author (s). This is an open-access article distributed under the terms of the Creative Commons Attribution 4.0 International License (CC-BY).

\section{Funding: None}

\section{Conflict of Interest: None}

\section{Keywords}

Double-blinded; randomized; planter fasciitis; platelet-rich plasma; ultrasound.

\section{References}

1. Gautham $P$, Nuhmani $S$, Kachanathu SJ. Plantar fasciitis-an update. Bangladesh Journal of Medical Science 2015;14(1):3-8.

2. Lareau CR, Sawyer GA, Wang JH, DiGiovanni CW. Plantar and medial heel pain: diagnosis and management. J Am Acad Orthop Surg 2014;22(6):372-380.

3. Martinelli N, Marinozzi A, Carnì S, Trovato U, Bianchi A, Denaro V. Platelet-rich plasma injections for chronic plantar fasciitis. Int Orthop 2013;37(5):839-842.

4. Owens BD, Wolf JM, Seelig AD, et al. Risk factors for lower extremity tendinopathies in military personnel. Orthop J Sports Med 2013;1(1); 2325967113492707.

5. Beeson P. Plantar fasciopathy: revisiting the risk factors. Foot Ankle Surg 2014;20(3):160-165.

6. Riddle DL, Schappert SM. Volume of ambulatory care visits and patterns of care for patients diagnosed with planter fasciitis: a national study of medical doctors. Foot Ankle Int 2004;25(5):303-310.

7. Schwartz EZ, Su J. Plantar fasciitis: a concise review. Perm J 2014; 18(1):e105-e107.

8. Buchbinder R. Clinical practice. Plantar fasciitis. N Eng/ J Med 2004 350(21):2159-2166.

9. Irving DB, Cook JL, Young MA, Menz HB. Impact of chronic plantar heel pain on health-related quality of life. J Am Podiatr Med Assoc 2008;98(4):283-289.

10. Covey CJ, Malder MD. Plantar fasciitis: how best to treat? J Fam Pract 2013;62(9):466-471.

11. Fitzpatrick J, Bulsara M, Zheng MH. The effectiveness of platelet- rich plasma in the treatment of tendinopathy: a meta-analysis of randomized controlled clinical trials. Am J Sports Med 2017;45(1):226233.

12. Bielecki T, Dohan Ehrenfest DM. Platelet-rich plasma (PRP) and 
platelet-Rich Fibrin (PRF): surgical adjuvents, preparations for in situ regenerative medicine and tools for tissue engineering. Curr pharm Biotechnol 2012;13(7):1121-1130.

13. Monto RR. Platelet-rich plasma and plantar fasciitis. Sports Med Arthrosc Rev 2013;21(4):220-224.

14. Cieslik-Bielecka A, Choukroun J, Odin G, Dohan Ehrenfest DM. L-PRP/L-PRF in esthetic plastic surgery, regenerative medicine of the skin and chronic wounds. Curr Pharm Biotechnol 2012;13(7):12661277.

15. Mehta S, Watson JT. Platelet rich concentrate; basic science and current clinical applications. J Orthop Trauma 2008;22(6):432-438.

16. Baz AA, Gad AM, Waly MR. Ultrasound guided injection of platelet rich plasma in cases of chronic plantar fasciitis. The Egyptian Journal of Radiology and Nuclear Medicine 2017;48:125-132.

17. Ragab EM, Othman AM. Platelets rich plasma for treatment of chronic plantar fasciitis. Arch Orthop Trauma Surg 2012;132(8):10651070.

18. Gupta SKV, Bandari D. Autologous platelet-rich plasma injection in tennis elbow and plantar fasciitis. Current Orthopaedic Practice 2016:27;405-408.

19. Mallah R El, Elattar EA, Zidan HF. Platelet-rich plasma versus dry needling of myofascial meridian trigger points in the treatment of plantar fasciitis. Egyptian Rheumatology \& Rehabilitation 2017:44;5868.

20. de Vos R, Weir A, van Schie HT, et al. Platelet-rich plasma injection for chronic achilles tendinopathy: a randomized controlled trial. JAMA 2010:303(2);144-149.

21. van Egmond JC, Breugem SJ, Driessen M, Bruijn DJ. Platelet-rich-plasma injection seems to be effective in treatment of plantar fasciitis: a case series. Acta Orthop Belg 2015;81(2):315-320.

22. Karabay N, Toros T, Hurel C. Ultrasonographic evaluation in plantar fasciitis. J Foot Ankle Surg 2007;46(6): 442-446.

23. Fabrikant JM, Park TS. Plantar fasciitis (fasciosis) treatment outcome study: correlated with patient self-reported improvement. Foot (Edinb) $2011 ; 21(2): 79-83$.

24. Mautner K, Malanga G, Colberg R. Optimization of ingredients, procedures and rehabilitation of platelet rich plasma injections for chronic tendinopathy. Pain Manag 2011;1(6):523-532.

25. Langberg $\mathrm{H}$, Ellingsgaard $\mathrm{H}$, Madsen $\mathrm{T}$, et al. Eccentric rehabilitation exercise increases peritendinous type I collagen synthesis in humans with Achilles tendinosis. Scand J Med Sci Sports 2007;17(1):6166.

26. Kjaer $\mathrm{M}$, Langberg $\mathrm{H}$, Heinemeier $\mathrm{K}$, et al. From mechanical loading to collagen synthesis, structural changes and function in human tendon. Scand J Med Sci Sports 2009;19(4):500-510.

27. Sheth U, Simunovic N, Klein G, et al. Efficacy of autologous platelet-rich plasma use for orthopaedic indications: a meta-analysis. J Bone Joint Surg Am 2012;94(4):298-307.

28. Butts R, Dunning J, Perreault T, Mourad F, Grubb M. Peripheral and spinal mechanisms of pain and dry needling mediated analgesia: a clinical resource guide for health care professionals. Int J Phys Med Rehabil 2016;4:327.

29. Chiew SK, Ramasamy TS, Amini F. Effectiveness and relevant factors of platelet- rich plasma treatment in managing plantar fasciitis: asystematic review. J Res Med Sci 2016;21:38. eCollection 2016.

30. Hamayouni K, Karimian H, Golkar HR, Jalalati N. Treatment of chronic plantar fasciitis with ultrasound-guided injection of platelets rich plasma. J Arch Mil Med 2016;4:e42332.

31. Schulhofer S. Short-term benefits of ultrasound-guided corticosteroid injection in plantar fasciitis. Clin J Sport Med 2013;23(1):83-84.

32. Kane D, Greaney T, Shanahan M, et al. The role of ultrasonography in the diagnosis and management of idiopathic plantar fasciitis. Rheumatology (Oxford) 2001;40(9):1002-1008.

33. Johnson-Lynn S, Cooney A, Ferguson D, et al. Pilot randomised con- trolled trial of platelet-rich plasma versus normal saline for plantar fasciitis. Orthopaedic Proceedings British Editorial Society of Bone \& Joint Surgery 2016;98:19-23.

34. Eftekharsadat B, Babaei-Ghazani A, Zeinolabedinzadeh V. Dry needling in patients with chronic heel pain due to plantar fasciitis: a single-blinded randomized clinical trial. Med J Islam Repub Iran 2016;30:401.

35. Cotchett MP, Landorf KB, Munteanu SE, Raspovic AM. Consensus for dry needling for plantar heel pain (plantar fasciitis): a modified Delphi study. Acupunct Med 2011;29(3):193-202.

36. Rastegar S, Baradaran Mahdavi S, Hoseinzadeh B, Badiei S. Comparison of dry needling and steroid injection in the treatment of plantar fasciitis: a single-blind randomized clinical trial. Int Orthop 2018;42(1):109-116.

37. Cotchett MP, Munteanu SE, Landorf KB. Effectiveness of trigger point dry needling for plantar heel pain: a randomized controlled trial. Phys Ther 2014:94(8);1083-1094.

38. Tahririan MA, Motififard M, Tahmasebi MN, Siavashi B. Planter fasciitis. J Res Med Sci 2012;17(8):799-804.

39. Filardo G, Kon E, Buda R, et al. Platelet-rich plasma intraarticular knee injections for the treatment of degenerative cartilage lesions and osteoarthritis. Knee Surg Sports Traumatol Arthrosc 2011;19(4):528535.

40. Kisiday JD, Mcllwraith CW, Rodkey WG, Frisbie DD, Steadman JR. Effects of platelet rich plasma composition on anabolic activities in equine cartilage and meniscal explants. Cartilage 2012;3(3):245-254.

41. De Candia E. Mechanisms of platelet activation by thrombin: a short history. Thromb Res 2012;129(3):250-256.

42. Filardo G, Kon E, Pereira Ruiz MT, et al. Platelet-rich plasma intra-articular injections for cartilage degeneration and osteoarthritis: single-versus double-spinning approach. Knee Surg Sports Traumatol Arthrosc 2011;20(10):2082-2091.

43. Bausset O, Giraudo L, Veran J, et al. Formulation and storage of platelet-rich plasma homemade product. Biores Open Access 2012;1(3):115-123.

44. Cavallo C, Roffi A, Grigolo B, et al. Platelet-rich plasma: the choice of activation method affects the release of bioactive molecules. BioMed Research International 2016:6591717;7.

45. Silva RF, Alvarez ME, Ríos DL, López C, Carmona JU, Rezende CM. Evaluation of the effect of calcium gluconate and bovine thrombin on the temporal release of transforming growth factor beta 1 and platelet-derived growth factor isoform BB from feline platelet concentrates. BMC Vet Res 2012;8:212.

46. Peerbooms JC, van Laar W, Faber F, Schuller HM, van der Hoeven $\mathrm{H}_{\text {, }}$ Gosens T. Use of platelet rich plasma to treat plantar fasciitis: Design of a multi centre randomized controlled trial. BMC Musculoskelet Disord 2010;11:69.

47. Graziani F, Ivanovski S, Cei S, Ducci F, Tonetti M, Gabriele M. The in vitro effect of different PRP concentrations on osteoblasts and fibroblasts. Clin Oral Implants Res 2006;17(2):212-219.

48. Castillo TN, Pouliot MA, Kim HJ, Dragoo JL. Comparison of growth factor and platelet concentration from commerical platelet-rich plasma separation systems. Am J Sports Med 2011;39(2):266-271.

49. Shekhar C, Jhan A, Singh G, et al. Role of leukocyte free platelet rich plasma in planter fasciitis: a prospective study. Int J Res Med Sci 2017;5(12):5432-5439.

50. Carofino B, Chowaniec DM, McCarthy MB, et al. Corticosteroids and local anesthetics decrease positive effects of platelet-rich plasma: an in vitro study on human tendon cells. Arthroscopy 2012;28(5):711719.

51. Kumar NV, Arvind Kumar SM, Udayamoorthy S, Kumar K. Platelet rich plasma in treatment of plantar fascitis. Int J Res Orthop 2017; 3(3):350-353.

52. Bausset O, Magalon J, Giraudo L, et al. Impact of local anaesthetics and needle calibers used for painless PRP injections on platelet functionality. Muscles Ligaments Tendons J 2014;4(1):18-23. 